Article

\title{
A New Open-framework Iron Borophosphate from Ionic Liquids: $\mathrm{KFe}\left[\mathrm{BP}_{2} \mathrm{O}_{8}(\mathrm{OH})\right]$
}

\section{Guangmei Wang and Anja-Verena Mudring *}

Fakultät für Chemie, Ruhr-Universität Bochum, D-44780 Bochum, Germany;

E-Mail: guangmei.wang@rub.de

* Author to whom correspondence should be addressed; E-Mail: anja.mudring@ rub.de;

Tel.: +49(0)234-32-27408 Fax: +49(0)234-32-14951.

Received: 11 April 2011; in revised form: 15 April 2011 / Accepted: 18 April 2011 /

Published: 19 April 2011

\begin{abstract}
A new open-framework iron borophosphate, $\mathrm{KFe}_{2}\left[\mathrm{BP}_{2} \mathrm{O}_{8}(\mathrm{OH})\right]$, has been obtained by ionothermal synthesis from $\mathrm{KH}_{2} \mathrm{PO}_{4}, \mathrm{FeCl}_{3} \cdot 4 \mathrm{H}_{2} \mathrm{O}, \mathrm{H}_{3} \mathrm{BO}_{3}$ and $\left[\mathrm{C}_{4}\right.$ mpyr $] \mathrm{Br}$ (1-butyl-1-methylpyrrolidinium bromide). Single-crystal $\mathrm{X}$-ray diffraction analysis shows that $\mathrm{KFe}\left[\mathrm{BP}_{2} \mathrm{O}_{8}(\mathrm{OH})\right]$ (monoclinic, $P 2_{1} / c, a=9.372(2) \AA, b=8.146(2) \AA, c=9.587(2) \AA$, $\beta=101.18(3)^{\circ}, V=718.0(2) \AA^{3}$ and $\left.Z=4\right)$ has a three-dimensional (3-D) framework structure composed by $\left\{\mathrm{Fe}(\mathrm{III}) \mathrm{O}_{5}(\mathrm{OH})\right\}$ octahedra as well as $\left\{\mathrm{BO}_{3}(\mathrm{OH})\right\}$ and $\left\{\mathrm{PO}_{4}\right\}$ tetrahedra. As anionic structural sub-unit, $\mathrm{KFe}\left[\mathrm{BP}_{2} \mathrm{O}_{8}(\mathrm{OH})\right]$, contains an infinite open-branched $\left\{\left[\mathrm{BP}_{2} \mathrm{O}_{8}(\mathrm{OH})\right]^{4-}\right\}$ chain which is formed by alternating $\left\{\mathrm{BO}_{3}(\mathrm{OH})\right\}$ and $\left\{\mathrm{PO}_{4}\right\}$ tetrahedra. $\left\{\mathrm{Fe}(\mathrm{III}) \mathrm{O}_{5}(\mathrm{OH})\right\}$ octahedra share common $\mathrm{O}$ corners with five phosphate tetrahedra and the $\mathrm{OH}$ corner links to the hydrogen borate group to give a $3 \mathrm{D}$ framework. The negative charges of the inorganic framework are balanced by $\mathrm{K}^{+}$ions.
\end{abstract}

Keywords: iron borophosphate; ionothermal synthesis; crystal structure

\section{Introduction}

Open-framework iron borophosphates have received much attention for their potential magnetic properties. A broad spectrum of borophosphates with various dimensionalities and stoichiometries has been prepared using hydro-/solvothermal, as well as boric acid flux methods with or without the 
employment of organic amines as templates [1-7]. So far, the ionothermal synthesis of iron borophosphates has not been explored [8-11].

The known borophosphate anionic frameworks can be derived from a few typical borophosphate fundamental building units (FBUs) with $\mathrm{B} / \mathrm{P}$ ratios of $6 / 1,5 / 1,3 / 1,3 / 2,1 / 1,3 / 4,2 / 3,1 / 2,2 / 5,1 / 3$ and $1 / 4$ [12,13]. Among them, nonameric FBUs, $\left[\mathrm{B}_{3} \mathrm{P}_{6} \mathrm{X}_{26}\right][14]$ and $\left[\mathrm{BP}_{2} \mathrm{X}_{8}\right][15](\mathrm{X}=\mathrm{O}, \mathrm{OH})$ with a $\mathrm{B} / \mathrm{P}$ ratio of $1 / 2$, form the largest group. Borophosphates of the general formula $A M\left[\mathrm{BP}_{2} \mathrm{O}_{8}(\mathrm{OH})\right](\mathrm{A}=\mathrm{K}$, $\mathrm{Rb}, \mathrm{Cs}$ or $\mathrm{NH}_{4}, \mathrm{M}=\mathrm{V}, \mathrm{Fe}, \mathrm{Al}, \mathrm{Ga}, \mathrm{Sc}$ ) have been described in the past several years [12]. Here we report on a new member of this family, $\mathrm{KFe}\left[\mathrm{B}\left(\mathrm{PO}_{4}\right)_{2} \mathrm{OH}\right]$, obtained by ionothermal synthesis.

\section{Results and Discussion}

$\mathrm{KFe}\left[\mathrm{BP}_{2} \mathrm{O}_{8}(\mathrm{OH})\right]$ was prepared by reacting ionothermally $\mathrm{FeCl}_{3} \cdot 4 \mathrm{H}_{2} \mathrm{O}, \mathrm{KH}_{2} \mathrm{PO}_{4}, \mathrm{H}_{3} \mathrm{BO}_{3}$ and 1-butyl-1-methylpyrrolidinium bromide, $\left[\mathrm{C}_{4} \mathrm{mpy}\right] \mathrm{Br}$, in a ratio of 3:3:1;1.5 at $200{ }^{\circ} \mathrm{C}$ for 5 days. Upon cooling crystals of $\mathrm{KFe}\left[\mathrm{BP}_{2} \mathrm{O}_{8}(\mathrm{OH})\right]$ formed. The crystal structure of $\mathrm{KFe}\left[\mathrm{BP}_{2} \mathrm{O}_{8}(\mathrm{OH})\right]$ (monoclinic, $P 2{ }_{1} / c, a=9.372(2) \AA, b=8.146(2) \AA, c=9.587(2) \AA, \beta=101.18(3)^{\circ}, V=718.0(2) \AA^{3}$ and $\left.Z=4\right)$ is built up by a 3-D framework of $\left\{\mathrm{Fe}(\mathrm{III}) \mathrm{O}_{6}\right\}$ octahedra, $\left\{\mathrm{BO}_{3}(\mathrm{OH})\right\}$ and $\left\{\mathrm{PO}_{4}\right\}$ tetrahedra. It is isotypic with $\mathrm{KAl}\left[\mathrm{BP}_{2} \mathrm{O}_{8}(\mathrm{OH})\right]$ [16], $\left(\mathrm{NH}_{4}\right) \mathrm{M}\left[\mathrm{BP}_{2} \mathrm{O}_{8}(\mathrm{OH})\right](\mathrm{M}, \mathrm{Al}, \mathrm{Ga}, \mathrm{Fe}, \mathrm{V})$ [17-20], RbM[BP $\mathrm{O}_{8}(\mathrm{OH})$ ] $(\mathrm{M}=\mathrm{Al}, \mathrm{Ga}, \mathrm{V}, \mathrm{Fe})$ [21-25], $\mathrm{Cs}\left[\mathrm{BP}_{2} \mathrm{O}_{8}(\mathrm{OH})\right](\mathrm{M}=\mathrm{Al}, \mathrm{Ga}, \mathrm{Fe})$ [26-28].

The asymmetric unit of $\mathrm{KFe}\left[\mathrm{BP}_{2} \mathrm{O}_{8}(\mathrm{OH})\right]$ contains one crystallographically distinct $\mathrm{Fe}^{3+}$ cation, one $\mathrm{B}^{3+}$ cation, one $\mathrm{K}^{+}$cation, two crystallographically distinct $\mathrm{P}^{5+}$ cations, which are all coordinated by oxygen (Figure 1).

Figure 1. Thermal ellipsoid plot (50\% probability) of the asymmetric unit of $\mathrm{KFe}\left[\mathrm{BP}_{2} \mathrm{O}_{8}(\mathrm{OH})\right]$.

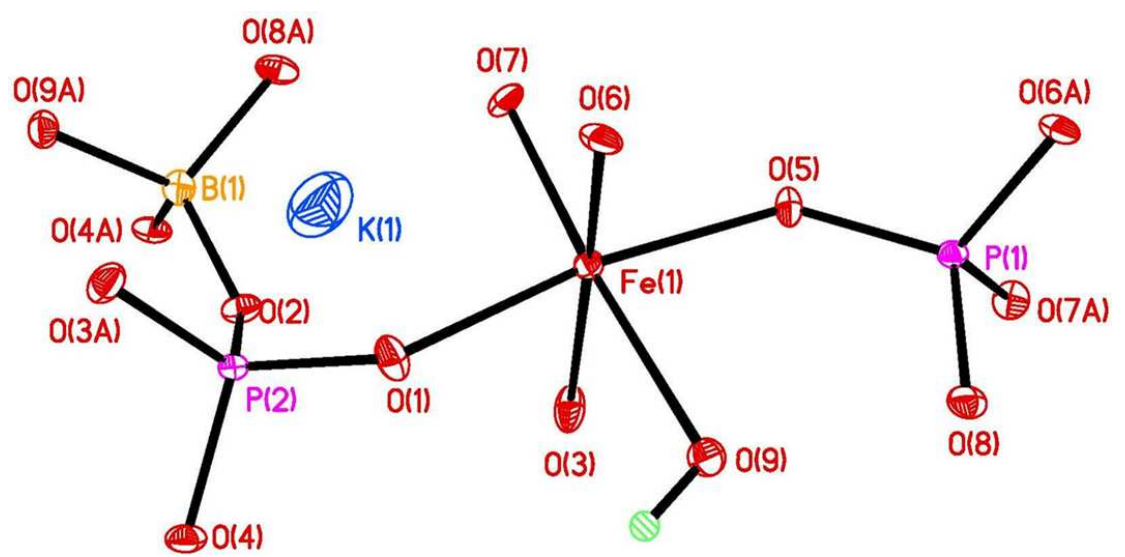

In $\mathrm{KFe}\left[\mathrm{BP}_{2} \mathrm{O}_{8}(\mathrm{OH})\right]$, the $\mathrm{Fe}(1)$ atom connects three $\mathrm{O}$ atoms to three $\mathrm{P}(1)$, two $\mathrm{O}$ atoms to two $\mathrm{P}(2)$ atoms, and one $\mathrm{O}(9) \mathrm{H}$ to the $\mathrm{B}(1)$ atom. The $\mathrm{Fe}-\mathrm{O}$ internuclear distances vary in the range of 1.954(4)-2.143(4) $\AA$. All boron and phosphorus atoms are tetrahedrally coordinated by oxygen atoms. The $\mathrm{P}(1)$ atom connects via three oxygen atoms to $\mathrm{Fe}(1)$ and one $\mathrm{O}$ atom to $\mathrm{B}(1)$ atom; the $\mathrm{P}(2)$ atom connects two $\mathrm{Fe}(1)$ atoms and two $\mathrm{B}(1)$ atoms via four oxygen atoms, respectively. The $\mathrm{P}-\mathrm{O}$ bond lengths vary in the range of $1.505(4)-1.562(3) \AA$. The $\mathrm{B}(1)$ atom connects via one oxygen atom to a $\mathrm{P}(1)$ atom, two oxygen atoms to $\mathrm{P}(2)$ atoms and one $\mathrm{O}(9) \mathrm{H}$ to $\mathrm{Fe}(1)$ atom, respectively. The $\mathrm{B}-\mathrm{O}$ bond lengths vary in the range of $1.457(6)-1.475(7) \AA$. 
Figure 2. a: Trimeric borophosphate basic building unit; b: open-branched vierer chain ${ }_{\infty}^{1}\left\{\left[\mathrm{BP}_{2} \mathrm{O}_{8}(\mathrm{OH})\right]^{4-}\right\} \mathrm{FBU}$; $\mathbf{c}$ : view of the open-framework structure of $\mathrm{KFe}\left[\mathrm{BP}_{2} \mathrm{O}_{8}(\mathrm{OH})\right]$ along the [001] direction showing irregular 8-ring channels (color code: Fe, green; $\mathrm{B}$, yellow; P, red; O, blue; K, purple; $\mathrm{H}$, white).
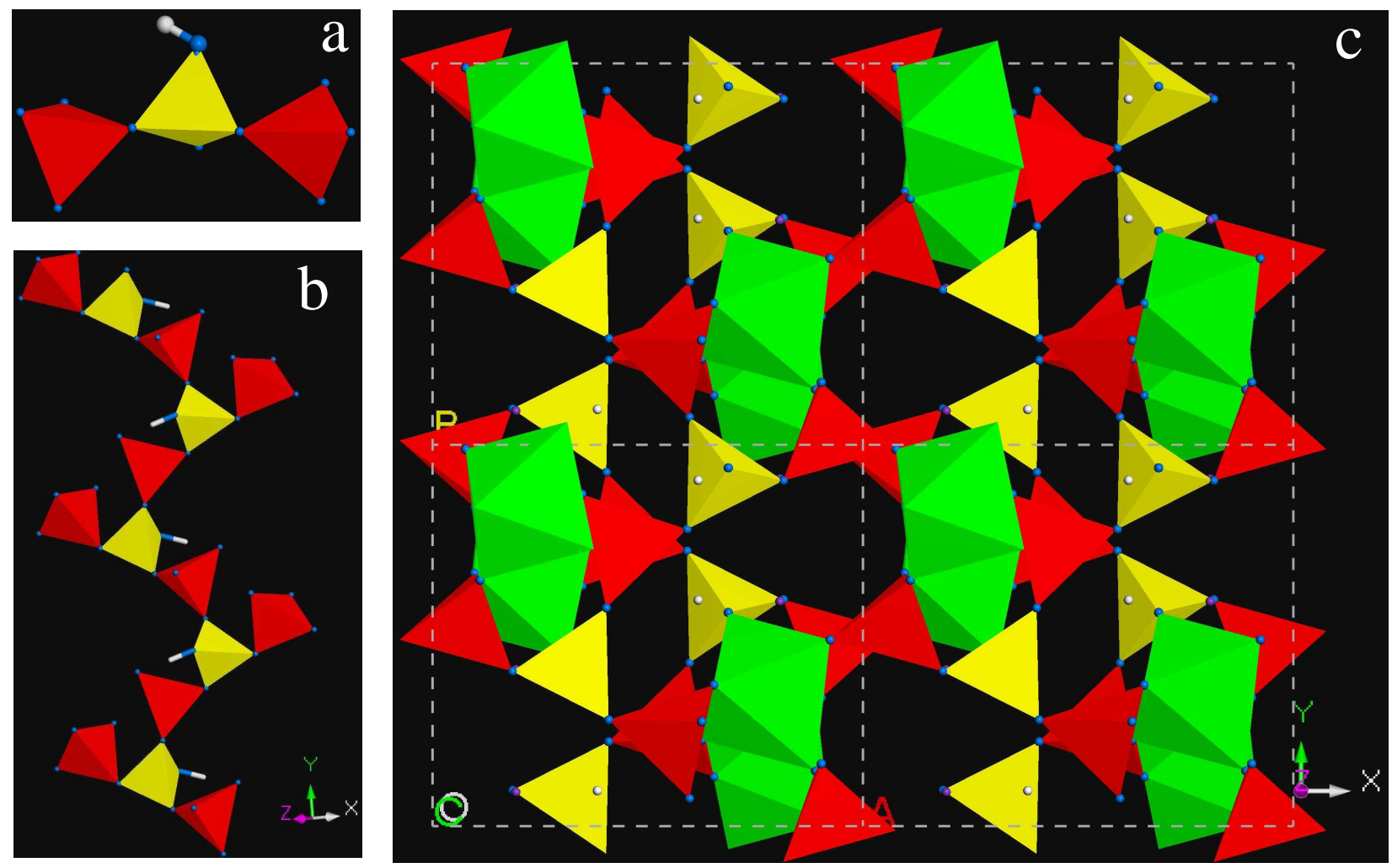

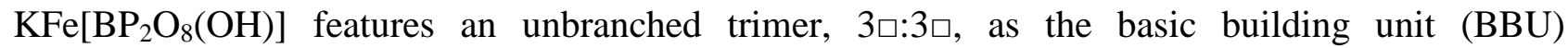
(Figure 2 a). These BBUs are connected to an infinite open-branched $(\mathrm{oB})$ vierer ${ }^{1}{ }_{\infty}\left[\left[\mathrm{BP}_{2} \Phi_{9}\right]_{2}\right](\Phi=\mathrm{O}$, $\mathrm{OH}$ ) chain (Figure $2 \mathrm{~b})$. The condensation of this borophosphate $\mathrm{FBU}$ with $\left\{\mathrm{Fe}(\mathrm{III}) \mathrm{O}_{5}(\mathrm{OH})\right\}$ octahedra by sharing common oxygen atoms with five phosphate tetrahedra and an $\mathrm{OH}$ group with a hydrogenborate group link to a 3-D framework (Figure 2c). It features 8-ring channels along the [100] direction enclosed by two $\mathrm{FeO}_{5}(\mathrm{OH})$ octahedra, two $\mathrm{BO}_{3}(\mathrm{OH})$ and four $\mathrm{PO}_{4}$ tetrahedra.

\section{Experimental Section}

$\mathrm{KFe}\left[\mathrm{BP}_{2} \mathrm{O}_{8}(\mathrm{OH})\right]$ is prepared under ionothermal synthesis conditions using the ionic liquid 1-butyl-1-methylpyrrolidinium bromide, $\left[\mathrm{C}_{4} \mathrm{mpyr}\right] \mathrm{Br}$, as the solvent. A mixture of $\mathrm{FeCl}_{3} \cdot 4 \mathrm{H}_{2} \mathrm{O}(99 \%$, Fluka), $\mathrm{KH}_{2} \mathrm{PO}_{4}$ (99\%, J.T. Baker), $\mathrm{H}_{3} \mathrm{BO}_{3}$ (99.8\%, Appl. Chem.) and [ $\left.\mathrm{C}_{4} \mathrm{mpy}\right] \mathrm{Br}$ (99\%, Merck) was reacted in a $3 \mathrm{~mL}$ Teflon-lined stainless steel container at $200{ }^{\circ} \mathrm{C}$ for 5 days followed by cooling to room temperature. The products were filtered off, washed with deionized water and acetone, filtered by suction, and dried at $60{ }^{\circ} \mathrm{C}$ for one day.

A suitable single crystal of $\mathrm{KFe}\left[\mathrm{BP}_{2} \mathrm{O}_{8}(\mathrm{OH})\right]$ was selected for single-crystal X-ray diffraction (XRD) analysis. The data were collected at ambient temperature using graphite-monochromated Mo-K $\alpha$ radiation on an Image Plate Diffraction System, IPDS I, (Stoe, Darmstadt, Germany). The data were corrected for Lorentz and polarization effects. Data correction was carried out with the program 
X-RED [29]. A face-indexed numerical absorption correction (X-SHAPE) was applied [30]. The structure was solved by direct methods and refined by full-matrix least-squares techniques with the SHELXTL crystallographic software package [31]. The Fe, B, P, and O atoms could be unambiguously located. The $\mathrm{K}^{+}$ions were subsequently located from a difference Fourier map. In the final refinement cycles $\mathrm{H}$ atoms associated with the hydroxyl groups were added computationally.

Further details of the crystal structure investigations may be obtained from the Fachinformationszentrum Karlsruhe, 76344 Eggenstein-Leopoldshafen, Germany (Fax: +49-7247-808666; E-Mail: crysdata@fiz-karlsruhe.de, http://www.fiz-karlsruhe.de/request_for_deposited_data.hmtl) on quoting ICSD-422900.

Crystal data for $\mathrm{KFe}\left[\mathrm{BP}_{2} \mathrm{O}_{8}(\mathrm{OH})\right]$, $\left(312.71 \mathrm{~g}^{-1} \mathrm{~mol}^{-1}\right)$; diffractometer IPDS-I, Stoe, Darmstadt; Mo-K $\mathrm{K}_{\alpha}$ (graphite monochromator, $\lambda=71.073 \mathrm{pm}$ ); $\mathrm{T}=293(2) \mathrm{K} ; 2 \theta_{\max }=56.18^{\circ} ; 100$ images, $0^{\circ} \leq \varphi \leq 200^{\circ} ; \Delta \varphi=2^{\circ}$; indices: $-12 \leq \mathrm{h} \leq 12,-10 \leq \mathrm{k} \leq 10,-12 \leq 1 \leq 12$; transmission (min, $\max )=0.0307,0.0929 ; \rho_{\text {calc }}=2.893 \mathrm{~g} \mathrm{~cm}^{-3} ; 8321$ reflection intensities measured of which 1726 were symmetrically independent, $\mathrm{R}_{\text {int }}=0.0653, \mathrm{~F}(000)=612, \mu=27.547 \mathrm{~mm}^{-1}$. Monoclinic, $\mathrm{P} 2{ }_{1} / c$ (no. 62), $a=9.372(2) \AA, b=8.146(2) \AA, c=9.587(2) \AA, \beta=101.18(3)^{\circ}, V=718.0(2) \AA^{3}$ and $Z=4$. R values: $\mathrm{R}_{1} / \mathrm{wR}_{2}$ for final indices with $\left[\mathrm{I}_{\mathrm{o}}>2 \sigma\left(\mathrm{I}_{\mathrm{o}}\right)\right]: 0.0400 / 0.1095$ and for all data: $0.0541 / 0.1122 ; \mathrm{S}_{\text {all }}=1.071$.

\section{Conclusions}

A new open-framework iron borophosphate $\mathrm{KFe}\left[\mathrm{BP}_{2} \mathrm{O}_{8}(\mathrm{OH})\right]$, has been prepared under ionothermal conditions using the ionic liquid 1-butyl-1-methyl pyrrolidinium bromide, $\left[\mathrm{C}_{4} \mathrm{mpy}\right] \mathrm{Br}$, as the solvent. The successful preparation of the new iron borophosphate in an ionic liquid demonstrates not only that many more open-framework borophosphates could be obtained, but also that the ionothermal method is a promising method to synthesize new kinds of open-framework materials.

\section{Acknowledgements}

This work is supported by the European Research Council (ERC) under the contract 200475.

\section{References and Notes}

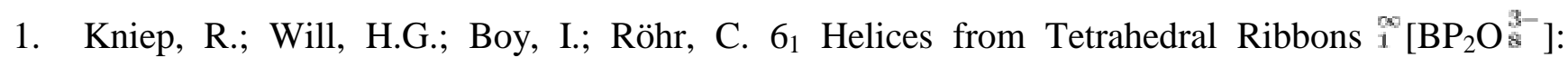
Isostructural Borophosphates $\mathrm{M}^{\mathrm{I}} \mathrm{M}^{\mathrm{II}}\left(\mathrm{H}_{2} \mathrm{O}\right)_{2}\left[\mathrm{BP}_{2} \mathrm{O}_{8}\right] \cdot \mathrm{H}_{2} \mathrm{O}\left(\mathrm{M}^{\mathrm{I}}=\mathrm{Na}, \mathrm{K} ; \mathrm{M}^{\mathrm{II}}=\mathrm{Mg}, \mathrm{Mn}, \mathrm{Fe}, \mathrm{Co}, \mathrm{Ni}\right.$, $\mathrm{Zn})$ and Their Dehydration to Microporous Phases $\mathrm{M}^{\mathrm{I}} \mathrm{M}^{\mathrm{II}}\left(\mathrm{H}_{2} \mathrm{O}\right)\left[\mathrm{BP}_{2} \mathrm{O}_{8}\right]$. Angew. Chem. Int. Ed. Engl. 1997, 36, 1013-1014.

2. Yang, T.; Li, G.B.; Ju, J.; Liao, F.H.; Xiong, M.; Lin, J.H. A series of borate-rich metalloborophosphates $\mathrm{Na}_{2}\left[\mathrm{M}^{\mathrm{II}} \mathrm{B}_{3} \mathrm{P}_{2} \mathrm{O}_{11}(\mathrm{OH})\right] \cdot 0.67 \mathrm{H}_{2} \mathrm{O}\left(\mathrm{M}^{\mathrm{II}}=\mathrm{Mg}, \mathrm{Mn}, \mathrm{Fe}, \mathrm{Co}, \mathrm{Ni}, \mathrm{Cu}, \mathrm{Zn}\right)$ : Synthesis, structure and magnetic susceptibility. J. Solid State Chem. 2006, 179, 2513.

3. Yilmaz, A.; Bu, X.H.; Kizilyalli, M.; Stucky, G.D. $\mathrm{Fe}\left(\mathrm{H}_{2} \mathrm{O}\right)_{2} \mathrm{BP}_{2} \mathrm{O}_{8} \cdot \mathrm{H}_{2} \mathrm{O}$, a First Zeotype Ferriborophosphate with Chiral Tetrahedral Framework Topology. Chem. Mater. 2000, 12, 3243-3245. 
4. Kniep, R.; Schäfer, G. Isotype Borophosphate $\mathrm{M}^{\mathrm{II}}\left(\mathrm{C}_{2} \mathrm{H}_{10} \mathrm{~N}_{2}\right)\left[\mathrm{B}_{2} \mathrm{P}_{3} \mathrm{O}_{12}(\mathrm{OH})\right]\left(\mathrm{M}^{\mathrm{II}}=\mathrm{Mg}, \mathrm{Mn}, \mathrm{Fe}, \mathrm{Ni}\right.$, $\mathrm{Cu}, \mathrm{Zn}$ ): Verbindungen mit Tetraeder-Schichtverbänden. Z. Anorg. Allg. Chem. 2000, 626, 141-147.

5. Huang, Y.X.; Ewald, B.; Schnelle, W.; Prots, Y.; Kniep, R. Chirality and Magnetism in a Novel Series of Isotypic Borophosphates: $\mathrm{M}^{\mathrm{II}}\left[\mathrm{BPO}_{4}(\mathrm{OH})_{2}\right]\left(\mathrm{M}^{\mathrm{II}}=\mathrm{Mn}, \mathrm{Fe}, \mathrm{Co}\right)$. Inorg. Chem. 2006, 45, 7578-7580.

6. Yang, W.T.; Li, J.Y.; Pan, Q.H.; Xing. H.H.; Chen, Y.; Yu, J.H.; Xu, R.R. Synthesis, structure and magnetic property of a new organo-templated mixed-valent iron(II, III) borophosphate. J. Mater. Chem. 2009, 19, 4523-4528.

7. Yang, T.; Sun, J.L.; Li, G.B.; Wang, Y.X.; Christensen, J.; He, Z.B.; Christensen, K.E.; Zou, X.D.; Liao, F.H; Lin, J.H. $\mathrm{Fe}_{5} \mathrm{O}_{5}\left[\mathrm{~B}_{6} \mathrm{O}_{10}(\mathrm{OH})_{3}\right] \cdot \mathrm{nH}_{2} \mathrm{O}$ : Wave-Layered Iron Borate and Frustrated Antiferromagnetism. Inorg. Chem. 2009, 48, 11209-11214.

8. Shi, H.; Shan, Y.; He, M.; Liu, Y. Impetus for solvothermal synthesis technique: Synthesis and structure of a novel 1-D borophosphate using ionic liquid as medium. J. Solid State Chem. 2003, 176, 33-36.

9. Lin, Z.; Wragg, D.S.; Lightfoot, P.; Morris, R.E. A novel non-centrosymmetric metallophosphateborate compound via ionothermal synthesis. Dalton Trans. 2009, 5287-5289.

10. Xing, H.; Li, Y.; Su, T.; Xu, J.; Yang, W.; Zhu, E.; Yu, J.; Xu, R. Spontaneous crystallization of a new chiral open-framework borophosphate in the ionothermal system. Dalton Trans. 2010, 39, 1713-1715.

11. Yang, M.; Xu, F.F.; Liu, Q.S.; Yan, P.F.; Liu, X.F.; Wang, C.; Welz-Biermann, U. Chelated orthoborate ionic liquid as a reactant for the synthesis of a new cobalt borophosphate containing extra-large 16-ring channels. Dalton Trans. 2010, 39, 10571-10573.

12. Kniep, R.; Engelhardt, H.; Hauf, C. A First Approach to Borophosphate Structural Chemistry. Chem. Mater. 1998, 10, 2930-2934, and references therein.

13. Ewald, B.; Huang, Y.X.; Kniep, R. Structural Chemistry of Borophosphates, Metalloborophosphates, and Related Compounds. Z. Anorg. Allg. Chem. 2007, 633, 1517-1540, and references therein.

14. Huang, Y.X.; Prots, Y.; Schnelle W.; Kniep, R. A new borophosphate chain anion in an organotemplated iron(III) borophosphate: Synthesis, crystal structure and magnetic properties of $\left(\mathrm{C}_{4} \mathrm{H}_{12} \mathrm{~N}_{2}\right)_{3} \mathrm{Fe}^{\mathrm{III}}{ }_{6}\left(\mathrm{H}_{2} \mathrm{O}\right)_{4}\left[\mathrm{~B}_{6} \mathrm{P}_{12} \mathrm{O}_{50}(\mathrm{OH})_{2}\right] \cdot 2 \mathrm{H}_{2} \mathrm{O}$. Sci. Technol. Adv. Mater. 2007, 8, 399-405.

15. Kniep, R.; Engelhardt, H. $\mathrm{Na}_{1.89} \mathrm{Ag}_{0.11}\left[\mathrm{BP}_{2} \mathrm{O}_{7}(\mathrm{OH})\right]$ und $\mathrm{Na}_{2}\left[\mathrm{BP}_{2} \mathrm{O}_{7}(\mathrm{OH})\right]$ - Isotype Borophosphate mit Tetraeder-Schichtpaketen. Z. Anorg. Allg. Chem. 1998, 624, 1291-1297.

16. Kniep, R.; Koch, D.; Hartmann, T. Crystal structure of potassium aluminum catena(monohydrogenmonoborate)-bis(monophosphate), $\mathrm{KAl}\left[\mathrm{BP}_{2} \mathrm{O}_{8}(\mathrm{OH})\right]$. Z. Kristallogr. NCS 2002, $217,186$.

17. Mi, J.; Huang, Y.X.; Deng, J.F.; Borrmann, H.; Zhao, J.T.; Kniep, R. Crystal structure of ammonium aluminum catena-[monohydrogenmonoborate-bis(monophosphate)], $\left(\mathrm{NH}_{4}\right) \mathrm{Al}$ $\left[\mathrm{BP}_{2} \mathrm{O}_{8}(\mathrm{OH})\right]$. Z. Kristallogr. NCS 2002, 217, 305-306. 
18. Li, M.R.; Mao, S.Y.; Huang, Y.X.; Mi, J.X.; Wei, Z.B.; Zhao, J.T.; Kniep, R. Crystal structure of ammonium gallium (monophosphate-hydrogenmonoborate-mono-phosphate), $\left(\mathrm{NH}_{4}\right) \mathrm{Ga}\left[\mathrm{BP}_{2} \mathrm{O}_{8}(\mathrm{OH})\right]$. Z. Kristallogr. NCS 2002, 217, 165-166.

19. Kriticos, M.; Wikstad, E.; Walldén, K. Hydrothermal synthesis, characterization and magnetic properties of three isostructural chain borophosphates; $\mathrm{NH}_{4} \mathrm{M}(\mathrm{III})\left[\mathrm{BP}_{2} \mathrm{O}_{8}(\mathrm{OH})\right]$ with $\mathrm{M}=\mathrm{V}$ or Fe and $\mathrm{NH}_{4}\left(\mathrm{Fe}(\mathrm{III})_{0.53} \mathrm{~V}(\mathrm{III})_{0.47}\right)\left[\mathrm{BP}_{2} \mathrm{O}_{8}(\mathrm{OH})\right]$. Solid State Sci. 2001, 3, 649-658.

20. Huang, Y.X.; Schäfer, G.; Carrillo Cabrera, W.; Cardoso, R.; Schnelle, W.; Zhao, J.T.; Kniep, R. Open-Framework Borophosphates: $\quad\left(\mathrm{NH}_{4}\right)_{0.4} \mathrm{Fe}_{0.55}^{\mathrm{II}} \mathrm{Fe}_{0.5}^{\mathrm{III}}\left(\mathrm{H}_{2} \mathrm{O}\right)_{2}\left[\mathrm{BP}_{2} \mathrm{O}_{8}\right]-0.6 \mathrm{H}_{2} \mathrm{O}$ and $\mathrm{NH}_{4} \mathrm{Fe}^{\mathrm{III}}\left[\mathrm{BP}_{2} \mathrm{O}_{8}(\mathrm{OH})\right]$. Chem. Mater. 2001, 13, 4348-4354.

21. Mi, J.; Zhao, J.T.; Huang, Y.X.; Deng, J.F.; Borrmann, H.; Kniep, R. Crystal structure of rubidium aluminum catena-[monohydrogen-monoborate-bis(monophosphate)], $\quad \operatorname{RbAl}\left[\mathrm{BP}_{2} \mathrm{O}_{8}(\mathrm{OH})\right] . \quad Z$. Kristallogr. NCS 2002, 217, 171-172.

22. Yakubovicha, O.V.; Steeleb, I.M.; Dimitrovab, O.V. Polymorphism of the Borophosphate Anion in $\mathrm{K}(\mathrm{Fe}, \mathrm{Al})\left[\mathrm{BP}_{2} \mathrm{O}_{8}(\mathrm{OH})\right]$ and $\mathrm{Rb}(\mathrm{Al}, \mathrm{Fe})\left[\mathrm{BP}_{2} \mathrm{O}_{8}(\mathrm{OH})\right]$ Crystal Structures. Kristallografiya 2010, $55,810-817$.

23. Mi, J.; Borrmann, H.; Mao, S.Y.; Huang, Y.-X.; Zhang, H.; Zhao, J.T.; Kniep, R. Crystal structure of rubidium gallium catena-[monohydrogen-monoborate-bis(monophosphate)] $\mathrm{RbGa}\left[\mathrm{BP}_{2} \mathrm{O}_{8}(\mathrm{OH})\right]$, from a twinned crystal. Z. Kristallogr. NCS 2003, 218, 17-18.

24. Engelhardt, H.; Borrmann, H.; Kniep, R. Crystal structure of rubidium vanadium(III) catena[monohydrogenmonoborate-bis(monophosphate)], $\left.\mathrm{RbV}_{\mathrm{BP}} \mathrm{O}_{8}(\mathrm{OH})\right]$. Z. Kristallogr. NCS 2000, 215, 203-204.

25. Kniep, R.; Boy, I.; Engelhard, H. $\mathrm{RbFe}\left[\mathrm{BP}_{2} \mathrm{O}_{8}(\mathrm{OH})\right]$ : A New Borophosphate Containing OpenBranched Tetrahedral Vierer-Einfach Chains. Z. Anorg. Allg. Chem. 1999, 625, 1512-1516.

26. Mi, J.; Huang, Y.X.; Deng, J.F.; Borrmann, H.; Zhao, J.T.; Kniep, R. Crystal structure of caesium aluminum catena-[monohydrogen-monoborate-bis(monophosphate)], $\quad \mathrm{CsAl}\left[\mathrm{BP}_{2} \mathrm{O}_{8}(\mathrm{OH}) \quad Z\right.$. Kristallogr. NCS 2002, 217, 169-170.

27. Mi, J.; Borrmann, H.; Huang, Y.X.; Mao, S.Y.; Zhao, J.T.; Kniep, R. Crystal structure of caesium gallium(III) catena-[monohydrogenmonoborate-bis(monophosphate)], $\quad \mathrm{CsGa}\left[\mathrm{BP}_{2} \mathrm{O}_{8}(\mathrm{OH})\right] \quad Z$. Kristallogr. NCS 2003, 218, 171-172.

28. Engelhard, H.; Kniep, R. Crystal structure of caesium iron(III) catena-[monohydrogenmonoboratebis(monophosphate)], CsFe[BP $\left.\mathrm{O}_{8}(\mathrm{OH})\right]$. Z. Kristallogr. NCS 1999, 214, 443-444.

29. X-RED 1.22, Stoe Data Reduction Program (C). Stoe \& Cie GmbH: Darmstadt, Germany, 2001.

30. X-Shape 1.06, Crystal Optimization for Numerical Absorption Correction (C). Stoe \& Cie GmbH: Darmstadt, Germany, 1999.

31. SHELXTL Program, version 5.1; Siemens Industrial Automation, Inc.: Madison, WI, USA, 1997.

(C) 2011 by the authors; licensee MDPI, Basel, Switzerland. This article is an open access article distributed under the terms and conditions of the Creative Commons Attribution license (http://creativecommons.org/licenses/by/3.0/). 